\title{
Visualization of Actin Organization and Quantification in Fixed Arabidopsis Pollen Grains and Tubes
}

Xiaolu $\mathrm{Qu}^{1}$, *, Qiannan Wang ${ }^{2}$, Haiyan Wang ${ }^{1}$ and Shanjin Huang ${ }^{2, *}$

\begin{abstract}
${ }^{1}$ Key Laboratory of Horticultural Plant Biology (Ministry of Education), College of Horticulture and Forestry Sciences, Huazhong Agricultural University, Wuhan, China; ${ }^{2}$ Center for Plant Biology, School of Life Sciences, Tsinghua University, Beijing 100084, China
\end{abstract}

*For correspondence: quxiaolu@mail.hzau.edu.cn; sjhuang@tsinghua.edu.cn

[Abstract] Although it is widely accepted that actin plays an important role in regulating pollen germination and pollen tube growth, how actin exactly performs functions remains incompletely understood. As the function of actin is dictated by its spatial organization, it is the key to reveal how exactly actin distributes in space in pollen cells. Here we describe the protocol of revealing and quantifying the spatial organization of actin using fluorescent phalloidin-staining in fixed Arabidopsis pollen grains and pollen tubes. We also introduce the method of assessing the stability and/or turnover rate of actin filaments in pollen cells using the treatment of latrunculin $B$.

Keywords: Arabidopsis, Pollen germination, Pollen grain, Pollen tube, Actin, Phalloidin staining

[Background] Pollen germination and subsequent pollen tube growth is the critical step during sexual plant reproduction, as it provides passage for the delivery of two non-motitle sperm cells to finally effect double fertilization in flowering plants (McCormick, 2013). How pollen germination and pollen tube growth are regulated has been subject to intensive scrutiny in the past several decades. It was shown that actin is an essential regulator of pollen germination and pollen tube growth and morphogenesis (Gibbon et al., 1999; Vidali et al., 2001). As pollen germination and pollen tube growth are very sensitive to the perturbation on the actin cytoskeleton (Gibbon et al., 1999; Vidali et al., 2001), and considering along with the fact that Arabidopsis thaliana is amenable to genetic analysis, Arabidopsis pollen has increasingly becoming the great cellular system in documenting the organization and regulation of the actin cytoskeleton toward understanding how actin performs functions in pollen $(\mathrm{Qu}$ et al., 2015). Arabidopsis pollen cell is an excellent cellular system to study the organization and regulation of the actin cytoskeleton is also because the spatial organization of actin is very regular in pollen cells (Ren and Xiang, 2007; Cheung and Wu, 2008; Chen et al., 2009; Staiger et al., 2010; Fu, 2015; Qu et al., 2015), and it is therefore comparatively easy to quantify the organization of actin in pollen cells (Fu, 2015; Qu et al., 2015). The colleagues in this field have developed different methods in revealing the organization of actin in pollen cells, including staining with fluorescent-phalloidin or immune-staining probed with anti-actin antibody in fixed Arabidopsis pollen cells as well as decoration with different actin markers in living pollen cells. Compared to immuno-staining with anti-actin antibody in fixed pollen cells, actin staining with fluorescent phalloidin can yield more cleanly filamentous images as phalloidin specifically binds to actin filaments with high affinity that reduces the background noise, 
which will facilitate subsequent analysis and quantification of actin organization in pollen cells. In addition, the procedure of actin staining with fluorescent phalloidin is comparatively simple and straightforward. Furthermore, the whole procedure was completed on solid pollen germination medium, which minimizes the effect of various treatments on the morphology of pollen tubes. This method has been carried out routinely and successfully in our laboratory. The detailed method can be found in series of our previously published papers (Wu et al., 2010; Zhang et al., 2010; Qu et al., 2013; Zheng et al., 2013; Chang and Huang, 2015; Liu et al., 2015; Zhang et al., 2016; Jiang et al., 2017; Qu et al., 2017; Lan et al., 2018; Diao et al., 2019; Jiang et al., 2019) and the papers from our colleagues in this community (Gebert et al., 2008; Cao et al., 2013; Jia et al., 2013; Zhu et al., 2017).

\section{Materials and Reagents}

A. Materials

1. $12 \mathrm{~cm} \times 12 \mathrm{~cm}$ square Petri dish

2. $9 \mathrm{~cm} \times 9 \mathrm{~cm}$ glass Petri dish (NORMAX, catalog number: 5058546)

3. Microscope slide

4. Parafilm (Merck, catalog number: P7668)

5. Glass bottom dish (In Vitro Scientific, catalog number: D35-20-1-N)

B. Plant materials: Arabidopsis thaliana Columbia-0 (Col-0)

Grow plants in culture room under $16 \mathrm{~h}$-light/8 h-dark photoperiod at $22^{\circ} \mathrm{C}$ (turn on light at 7:00 am and turn off light at 11:00 pm). Choose plants in the best flower production stage (i.e., choose plants with over 5 siliques on the main inflorescence stem, and don't choose older plant with few flower buds) (Figure 1A). 

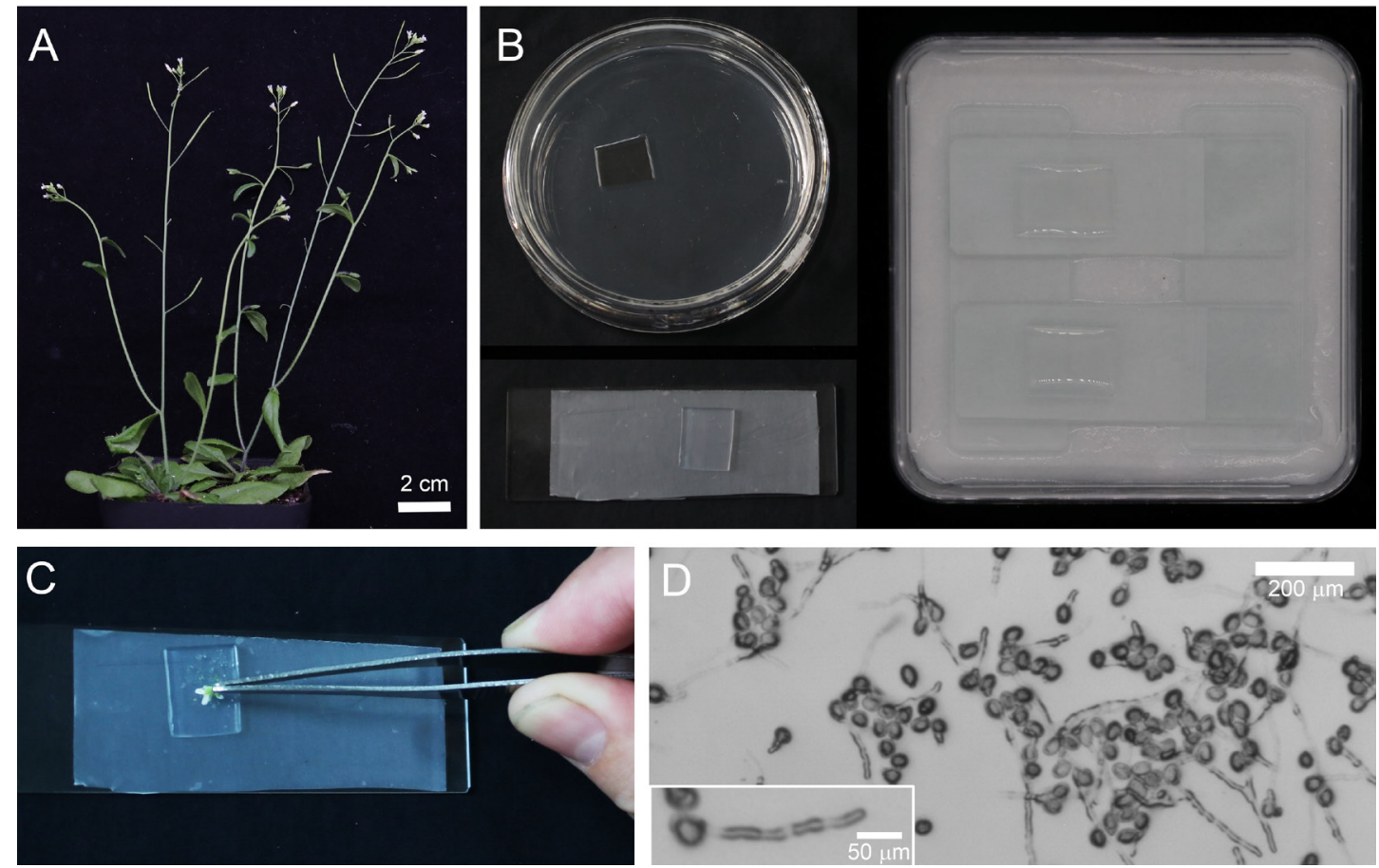

Figure 1. The Procedure for Germinating Arabidopsis Pollen on Solid Pollen Germination Medium. A. 6-8 weeks old Arabidopsis with flowers ready for the collection of pollen. Scale bar $=2$ $\mathrm{cm}$. B. Preparation of pollen germination medium and humid dish for pollen germination. C. Gently dip flowers to spread pollen on the surface of solid pollen germination medium. D.Images of pollen tubes after culture for $2 \mathrm{~h}$ at $28{ }^{\circ} \mathrm{C}$. Scale bar $=200 \mu \mathrm{m}$. Inset is a pollen tube with the length reaches at $\sim 180 \mu \mathrm{m}$. Scale bar $=50 \mu \mathrm{m}$. Pollen tubes are ready for subsequent fixation and staining with phalloidin.

C. Chemical reagents

1. Calcium chloride (Merck/Sigma-Aldrich, catalog number: C7902)

2. Calcium nitrate (Merck/Sigma-Aldrich, catalog number: C1396)

3. Magnesium sulfate (Merck/Sigma-Aldrich, catalog number: M1880)

4. Boric acid (Merck/Sigma-Aldrich, catalog number: B6768)

5. Sucrose (Merck/Sigma-Aldrich, catalog number: V900116)

6. Agarose (Merck/Sigma-Aldrich, catalog number: V900510)

7. Potassium hydroxide (Merck/Sigma-Aldrich, catalog number: 484016)

8. LatrunculinB (LatB) (Merck/Calbiochem, catalog number: 428020)

9. Dimethyl sulfoxide (DMSO) (Merck/Sigma-Aldrich, catalog number: D2438)

10. 3-Maleimidobenzoic acid N-hydroxysuccinimide ester (MBS) (Merck/Sigma-Aldrich, catalog number: M2786)

11. Trizma (Merck/Calbiochem, catalog number: V900483)

12. Sodium chloride (Merck/Sigma-Aldrich, catalog number: V900058)

13. Nonidet P-40 (NP-40) (IGEPAL CA-630) (Merck/Sigma-Aldrich, catalog number: I-8896)

14. Alexa Fluor 488 phalloidin (Thermo/Invitrogen, catalog number: A12379) 
15. Methanol (Merck/Sigma-Aldrich, catalog number: 494437)

16. Milli-Q water (Merck)

17. Pollen germination medium (see Recipes)

18. Tris Buffered Saline (TBS) (see Recipes)

19. Stock solution (see Recipes)

20. Individual stocks with Milli-Q water (see Recipes)

\section{Equipment}

1. Olympus FV1000S or an equivalent confocal microscope

2. Olympus $\mathrm{CX} 23$ or an equivalent microscope

3. Yiheng GHP-9050 or an equivalent constant temperature incubator

4. $4{ }^{\circ} \mathrm{C}$ and $-20^{\circ} \mathrm{C}$ freezer

\section{Software}

1. ImageJ (https://imagej.nih.gov/ij/ version 1.52n)

2. R program (http://www.r-project.org)

\section{Procedure}

A. Preparation of Arabidopsis pollen germination medium (Figure 1B)

1. Prepare individual stocks with Milli-Q water (Recipe 1).

2. Prepare $100 \mathrm{ml}$ Arabidopsis liquid pollen germination medium (PGM) (Recipe 2). Transfer $30 \mathrm{ml}$ liquid PGM into another conical flask to prepare solid PGM. Melting agarose with microwave, and evenly spread the medium into two or three $9 \mathrm{~cm} \times 9 \mathrm{~cm}$ glass Petri dishes. Notes:

a. $\mathrm{pH}$ at 6.85-7.0 works. Avoid adding excess $\mathrm{KOH}$.

b. $P G M$ can be used within 3 days under $4{ }^{\circ} \mathrm{C}$. Old PGM will dramatically reduce germination rate.

c. Remember to record the weight of the PGM before and after melting agarose. The amount of evaporated water can be quantified by the difference between the two records. Add Milli-Q water back to substitute the evaporated water.

d. Make sure the cooling surface for placing the Petri dishes is horizontal.

3. Cut solid PGM into $1 \mathrm{~cm} \times 1 \mathrm{~cm}$ pieces. Place the medium pieces onto parafilm, and move medium pieces and parafilm together onto microscope slide.

4. Put the microscope slide into humid $12 \mathrm{~cm} \times 12 \mathrm{~cm}$ square Petri dish. Place two microscope slides below to avoid water contamination. 
B. Pollen collection and pollen germination

1. Harvest fresh opened Arabidopsis flowers during the period from 9:30 am to 12:00 am and leave them at room temperature. Store no longer than $1 \mathrm{~h}$.

2. Gently dipping the Arabidopsis flower onto the medium to spread the pollen (Figure 1C). For the observation of pollen grains, collect pollen grains from over 20 Arabidopsis flowers in order to get a higher density. For the observation of pollen tubes, collect pollen grains from 10 fresh Arabidopsis flowers will be enough.

3. To germinate pollen, incubate the medium at $28{ }^{\circ} \mathrm{C}$ for $2 \mathrm{~h}$. The pollen tube is suitable for further observation or subject to fixation at about $200 \mu \mathrm{m}$ (Figure 1D).

C. Actin staining with phalloidin conjugated to fluorescent dye (Video 1)

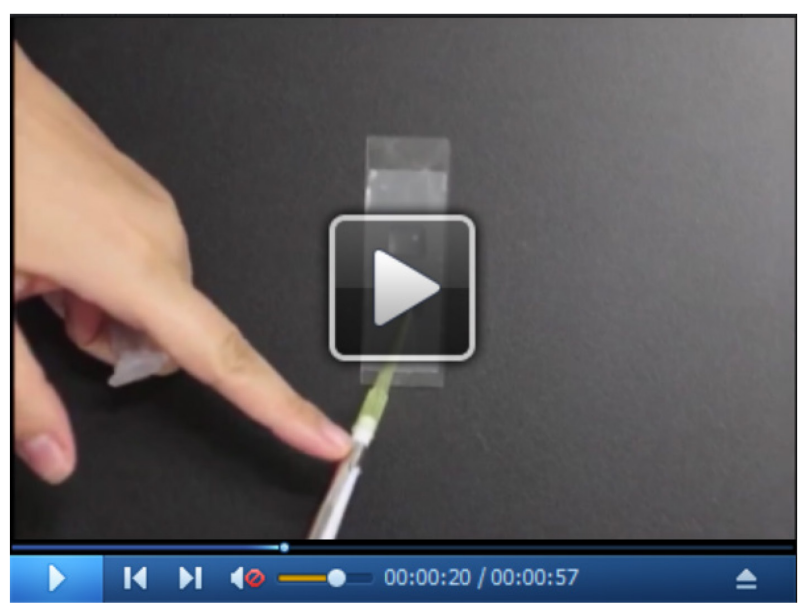

Video 1. Typical procedure for pollen grain and pollen tube fixation and actin staining

1. Several chemicals were dissolved in solvent according to the product specification and store as stock solution (Recipe 3).

2. To treat pollen cells with LatB, dilute LatB into liquid PGM to certain concentrations, e.g., 150 $\mathrm{nM}$ or $100 \mathrm{nM}$. Considering that actin structures in pollen tube are more sensitive to the application of LatB than that in pollen grains, usually, $100 \mathrm{nM}$ LatB was applied to pollen tube and $150 \mathrm{nM}$ LatB was applied to pollen grains. After incubation with LatB for $30 \mathrm{~min}$, pollen cells were subjected to fixation with MBS. DMSO in PGM was used as the control.

3. Fix the pollen cells with $300 \mu \mathrm{M}$ MBS for $1 \mathrm{~h}$ at $28^{\circ} \mathrm{C}$. Adding $100 \mu \mathrm{l} \mathrm{MBS-PGM}$ for each slice.

4. Remove MBS-PGM and penetrate the pollen cells by $0.05 \%$ NP- $40,150 \mu \mathrm{M}$ MBS in PGM for $10 \mathrm{~min}$.

5. Wash samples with TBS $+0.05 \%$ NP40 (Recipe 4) for three times. Keep $100 \mu \mathrm{l}$ TBS $+0.05 \%$ NP40 on samples for 10 min each time.

6. Stain samples with $200 \mathrm{nM}$ Alexa Fluor 488 phalloidin in TBS over night at $4{ }^{\circ} \mathrm{C}$ in humid square Petri dish. $30 \mu$ phalloidin buffer is enough for each slice.

7. Wash samples before observation with TBS once. 
Notes:

a. The concentration of DMSO in the PGM should be below $1 \%$.

b. Always pay attention to the morphology of the samples. Burst of pollen grain, swell pollen tube tip, and burst of pollen tube, may indicate the inadequacy of buffer.

c. Replace new parafilm at Step C6 can avoid buffer slipping from the solid PGM.

d. Avoid exposure dye to bright light all the time. Cover square Petri dish in tin foil after adding dye.

D. Visualization of actin filaments with laser scanning confocal microscope

1. Mount the solid PGM pieces to glass bottom dish to image with Olympus FV1000MPE multiphoton laser scanning confocal microscope equipped with a 100x objective (numerical aperture of 1.4).

2. Excite samples with $488 \mathrm{~nm}$ argon laser and collect emission light at 505-605 nm wavelength. Zoom pollen grain and pollen tube by $2 x$, image optical slice at $0.5 \mu \mathrm{m}$ step size.

Note: For image quantification, acquisition parameters must be identical in all images. Avoid overexposure.

\section{Data analysis}

To quantify the amount and organization of actin filaments in pollen tubes and pollen grains, the representative results can be found in Figure 2 .

1. Import raw images (i.e., oib files for Olympus microscope) into ImageJ software (http://imagej.nih.gov/ii]; version 1.52n). Filamentous structures can be distinguished in individual optical slice (Figure 2Aa, Figure 2Ba).

2. Quantify the fluorescent intensity to determine the relative amount of filamentous actin in pollen grains and pollen tubes. Perform the maximum intensity projection of the Z-stack images by Image " $Z$ project" function (Figure $2 \mathrm{Ab}$, Figure $2 \mathrm{Bb}$, Figure $2 \mathrm{Cb}$ ). Select a $5 \mu \mathrm{m} \times 10 \mu \mathrm{m}$ region at the tip of pollen tube by "Specify" function under "Selection" menu (Figure 2Ab). Select the whole region of pollen grain with "Polygon selection" function (Figure 2Bb, Figure $2 \mathrm{Cb}$ ). Compared to actin filaments in untreated pollen grains, actin filaments became fragmented and depolymerized under LatB treatment (Figures $2 \mathrm{Ca}-2 \mathrm{Cb}$ ). Measure the mean gray value of the selected region from over 20 pollen tubes or over 30 pollen grains to obtain the average fluorescent intensity. The amount of actin filaments can be quantified via measuring the fluorescent intensity, and it is significantly reduced in LatB treated pollen grains (Figure 2D). Statistical analysis was performed by the Student's $t$-test with $\mathrm{R}$ program (http://www.r-project.org).

3. Quantify the filament length and angles formed between actin cables and the pollen tube growth axis by "line" tool to analyze the actin structure. Do "maximum intensity projection" with adjacent 3 or 4 slices to facilitate the distinguishment of individual filaments. Measure angle 
and length in slices of the semi-projected images. Plot data and do kernel density estimation with R program (http://www.r-project.org) (Figures 2Ac-2Ad).
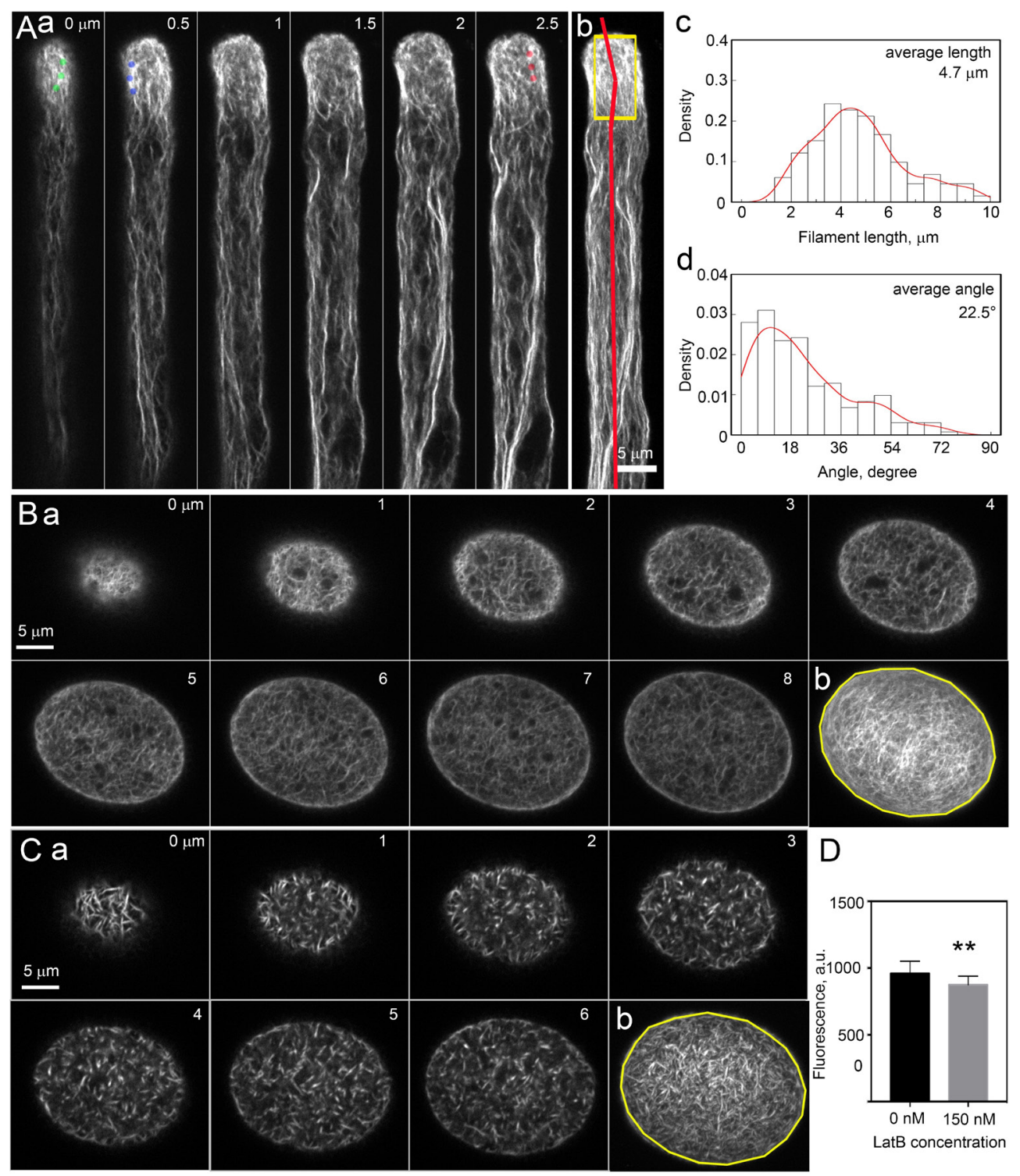

Figure 2. Observation and Quantification of Actin Structures in Pollen Tube and Pollen

Grain. A. a), Optical Z-slice images of a pollen tube, with $0.5 \mu \mathrm{m}$ z-step-size. Individual actin filaments can be distinguished in slices, which were marked by green, blue, and red dots, respectively. A. b), Maximum intensity projected pollen tube shown in (a). Fluorescent intensity can be measured from this kind of images in order to indicate the relative amount of filamentous actin. The yellow box indicates actin filaments at the apex and subapex of the pollen tube. The grow axis of the pollen tube is indicated by red line. Scale bar $=5 \mu \mathrm{m}$. A. c), Histogram of the length of actin filaments in yellow boxed region. A. d), Histogram of the angles formed between actin filaments and the growth axis in yellow boxed region. B. a), Optical Z-slice images of a pollen grain, with $1 \mu \mathrm{m}$ z-step-size. B. b), Maximum intensity projected pollen grain in (a). Fluorescent intensity in yellow circle region will be measured. Scale bar $=5$ 
$\mu \mathrm{m}$. C. a), Optical Z-slice images of a pollen grain after being subjected to the treatment with $150 \mathrm{nM}$ LatB. The images were displayed with z-step-size at $1 \mu \mathrm{m}$. C. b), Maximum intensity projected pollen grain shown in (a). Fluorescent intensity of phalloidin staining within yellow circle region was measured. Scale bar $=5 \mu \mathrm{m}$. D. Quantification of the fluorescent intensity in LatB treated and untreated pollen grains. $n=30,{ }^{* *} P<0.01$ by Student's $t$-test.

\section{$\underline{\text { Recipes }}$}

1. Individual stocks with Milli-Q water

$$
\begin{aligned}
& 100 \mathrm{mM} \mathrm{CaCl}_{2} \\
& 100 \mathrm{mM} \mathrm{Ca}\left(\mathrm{NO}_{3}\right)_{2} \\
& 100 \mathrm{mM} \mathrm{MgSO}_{4} \\
& 1 \% \mathrm{H}_{3} \mathrm{BO}_{3}(\mathrm{wt} / \mathrm{vol})
\end{aligned}
$$

Keep stocks at $4{ }^{\circ} \mathrm{C}$ and use within 6 months

2. Pollen germination medium

$1 \mathrm{mM} \mathrm{CaCl}_{2}$

$1 \mathrm{mM} \mathrm{Ca}\left(\mathrm{NO}_{3}\right)_{2}$

$1 \mathrm{mM} \mathrm{MgSO}_{4}$

$0.01 \% \mathrm{H}_{3} \mathrm{BO}_{3}$ (wt/vol)

$18 \%$ (wt/vol) sucrose

Adjust $\mathrm{pH}$ to $6.85-7.0$ with $\mathrm{KOH}$

For solid PGM, add $0.8 \%$ agarose

3. Stock solution

$100 \mathrm{mM}$ MBS (in DMSO)

$5 \mathrm{mM}$ LatB (in DMSO)

$6.6 \mu \mathrm{M}$ Alexa Fluor 488 phalloidin (in methanol)

Aliquote and store them at $-20^{\circ} \mathrm{C}$ freezer in order to avoid repeated freeze-thaw cycles

4. Tris Buffered Saline (TBS)

$50 \mathrm{mM}$ Tris (diluted from $1 \mathrm{M}$ stock, $\mathrm{pH}$ 7.4)

$200 \mathrm{mM} \mathrm{NaCl}$

$400 \mathrm{mM}$ Sucrose

\section{Acknowledgments}

This work was supported by grants from national natural science foundation of China (31671390 and 31970180) and the startup fund from Huazhong Agricultural University. This protocol was adapted from previous published work (Wu et al., 2010; Zhang et al., 2010). 


\section{Competing interests}

The authors declared that they have no conflicts of interest to this work.

\section{$\underline{\text { References }}$}

1. Cao, L. J., Zhao, M. M., Liu, C., Dong, H. J., Li, W. C. and Ren, H. Y. (2013). LISR28 is involved in pollen germination by affecting filamentous actin dynamics. Mol Plant 6(4): 1163-1175.

2. Chang, M. and Huang, S. (2015). Arabidopsis ACT11 modifies actin turnover to promote pollen germination and maintain the normal rate of tube growth. Plant $J$ 83(3): 515-527.

3. Chen, N., Qu, X., Wu, Y. and Huang, S. (2009). Regulation of actin dynamics in pollen tubes: control of actin polymer level. J Integr Plant Biol 51(8): 740-750.

4. Cheung, A. Y. and Wu, H. M. (2008). Structural and signaling networks for the polar cell growth machinery in pollen tubes. Annu Rev Plant Biol 59: 547-572.

5. Diao, M., Li, X. and Huang, S. (2019). Arabidopsis AlP1-1 regulates the organization of apical actin filaments by promoting their turnover in pollen tubes. Sci China Life Sci.

6. Fu, Y. (2015). The cytoskeleton in the pollen tube. Curr Opin Plant Biol 28: 111-119.

7. Gebert, M., Dresselhaus, T. and Sprunck, S. (2008). F-actin organization and pollen tube tip growth in Arabidopsis are dependent on the gametophyte-specific Armadillo repeat protein ARO1. Plant Cell 20(10): 2798-2814.

8. Gibbon, B. C., Kovar, D. R. and Staiger, C. J. (1999). Latrunculin B has different effects on pollen germination and tube growth. Plant Cell 11(12): 2349-2363.

9. Jia, H., Li, J., Zhu, J., Fan, T., Qian, D., Zhou, Y., Wang, J., Ren, H., Xiang, Y. and An, L. (2013). Arabidopsis CROLIN1, a novel plant actin-binding protein, functions in cross-linking and stabilizing actin filaments. J Biol Chem 288(45): 32277-32288.

10. Jiang, Y., Chang, M., Lan, Y. and Huang, S. (2019). Mechanism of CAP1-mediated apical actin polymerization in pollen tubes. Proc Natl Acad Sci U S A 116(24): 12084-12093.

11. Jiang, Y., Wang, J., Xie, Y., Chen, N. and Huang, S. (2017). ADF10 shapes the overall organization of apical actin filaments by promoting their turnover and ordering in pollen tubes. $J$ Cell Sci 130(23): 3988-4001.

12. Lan, Y., Liu, X., Fu, Y. and Huang, S. (2018). Arabidopsis class I formins control membrane-originated actin polymerization at pollen tube tips. PLoS Genet 14(11): e1007789.

13. Liu, X., Qu, X., Jiang, Y., Chang, M., Zhang, R., Wu, Y., Fu, Y. and Huang, S. (2015). Profilin regulates apical actin polymerization to control polarized pollen tube growth. Mol Plant 8(12): 1694-1709.

14. McCormick, S. (2013). Pollen. Curr Biol 23(22): R988-R990.

15. Qu, X., Jiang, Y., Chang, M., Liu, X., Zhang, R. and Huang, S. (2015). Organization and regulation of the actin cytoskeleton in the pollen tube. Front Plant Sci 5: 786.

16. Qu, X., Zhang, H., Xie, Y., Wang, J., Chen, N. and Huang, S. (2013). Arabidopsis villins 
promote actin turnover at pollen tube tips and facilitate the construction of actin collars. Plant Cell 25(5): 1803-1817.

17. Qu, X., Zhang, R., Zhang, M., Diao, M., Xue, Y. and Huang, S. (2017). Organizational innovation of apical actin filaments drives rapid pollen tube growth and turning. Mol Plant 10(7): 930-947.

18. Ren, H. and Xiang, Y. (2007). The function of actin-binding proteins in pollen tube growth. Protoplasma 230(3-4): 171-182.

19. Staiger, C. J., Poulter, N. S., Henty, J. L., Franklin-Tong, V. E. and Blanchoin, L. (2010). Regulation of actin dynamics by actin-binding proteins in pollen. $J$ Exp Bot 61(7): 1969-1986.

20. Vidali, L., McKenna, S. T. and Hepler, P. K. (2001). Actin polymerization is essential for pollen tube growth. Mol Biol Cell 12(8): 2534-2545.

21. Wu, Y., Yan, J., Zhang, R., Qu, X., Ren, S., Chen, N. and Huang, S. (2010). Arabidopsis FIMBRIN5, an actin bundling factor, is required for pollen germination and pollen tube growth. Plant Cell 22(11): 3745-3763.

22. Zhang, H., Qu, X., Bao, C., Khurana, P., Wang, Q., Xie, Y., Zheng, Y., Chen, N., Blanchoin, L., Staiger, C. J. and Huang, S. (2010). Arabidopsis VILLIN5, an actin filament bundling and severing protein, is necessary for normal pollen tube growth. Plant Cell 22(8): 2749-2767.

23. Zhang, M., Zhang, R., Qu, X. and Huang, S. (2016). Arabidopsis FIM5 decorates apical actin filaments and regulates their organization in the pollen tube. J Exp Bot 67(11): 3407-3417.

24. Zheng, Y., Xie, Y., Jiang, Y., Qu, X. and Huang, S. (2013). Arabidopsis actin-depolymerizing factor7 severs actin filaments and regulates actin cable turnover to promote normal pollen tube growth. Plant Cell 25(9): 3405-3423.

25. Zhu, J., Nan, Q., Qin, T., Qian, D., Mao, T., Yuan, S., Wu, X., Niu, Y., Bai, Q., An, L. and Xiang, Y. (2017). Higher-ordered actin structures remodeled by Arabidopsis ACTIN-DEPOLYMERIZING FACTOR5 Are important for pollen germination and pollen tube growth. Mol Plant 10(8): 1065-1081. 